\title{
Survival of Exfoliated Epithelial Cells: A Delicate Balance between Anoikis and Apoptosis
}

\author{
Bertrand Kaeffer \\ UMR-Phan 1280, Place Alexis Ricordeau, 44000 Nantes, France \\ Correspondence should be addressed to Bertrand Kaeffer, bertrand.kaeffer@univ-nantes.fr \\ Received 27 June 2011; Accepted 22 August 2011 \\ Academic Editor: M. Piacentini \\ Copyright () 2011 Bertrand Kaeffer. This is an open access article distributed under the Creative Commons Attribution License, \\ which permits unrestricted use, distribution, and reproduction in any medium, provided the original work is properly cited.
}

The recovery of exfoliated cells from biological fluids is a noninvasive technology which is in high demand in the field of translational research. Exfoliated epithelial cells can be isolated from several body fluids (i.e., breast milk, urines, and digestives fluids) as a cellular mixture (senescent, apoptotic, proliferative, or quiescent cells). The most intriguing are quiescent cells which can be used to derive primary cultures indicating that some phenotypes retain clonogenic potentials. Such exfoliated cells are believed to enter rapidly in anoikis after exfoliation. Anoikis can be considered as an autophagic state promoting epithelial cell survival after a timely loss of contact with extracellular matrix and cell neighbors. This paper presents current understanding of exfoliation along with the influence of methodology on the type of gastrointestinal epithelial cells isolated and, finally, speculates on the balance between anoikis and apoptosis to explain the survival of gastrointestinal epithelial cells in the environment.

\section{Introduction}

The recovery of exfoliated cells from biological fluids is a non-invasive technology which is in high demand in the field of translational research as well as during long-term experiments designed to minimize the sacrifice of long-lived or precious animals. Exfoliated epithelial cells can be used as surrogate for tissue biopsies in predicting changes in gene expression, DNA methylation, DNA damage, protein expression, and accumulation of dietary components [1,2]. Exfoliation has also been described as an active biochemical process linked to the homeostasis of gut epithelium [3-6]. It is believed that epithelial cells, loosing contact with companion cells (like fibroblasts) as well as extracellular matrix, enter anoikis [7]. Recent in vitro models are opening new avenues to conceptualize the exfoliation of gut epithelia in order to explain this highly context-dependent phenomenon. Loss of extracellular matrix contact induces autophagy in normal epithelial cells, and autophagy promotes the survival of detached cells during both anoikis and lumen formation in $3 \mathrm{D}$ epithelial cell culture $[8,9]$. Under these assumptions, exfoliation may be understood as a natural process to remove external cells from the luminal surface of an epithelium. Consequently, exfoliation may have a physiological role by allowing the formation of a lumen, preserving the epithelium's architecture, and, we can surmise, by providing sufficient flexibility to preserve the physical integrity of epithelia and allow its growth. In three-dimensional epithelial cell cultures, both autophagy and apoptosis are observed during lumen formation $[8,9]$. By loosing contact with the original mucosa, exfoliated epithelial cells have to activate autophagy as a survival mechanism to endure starvation. Starving cells are degrading cytoplasmic material to generate both nutrients and energy [10]. Indeed, quiescent exfoliated epithelial cells without signs of apoptosis can be recovered under specific clinical conditions in gastric fluid aspirates [4] or by suction from breast glands $[11,12]$ or extensive rinsing at the end of routine colonoscopy [13]. Many exfoliated quiescent epithelial cells can be cultured suggesting that detachmentinduced autophagy contributes to the viability of these cells. However, the survival of quiescent epithelial cells outside the tissue structure is highly variable. Human mammary epithelial cells die after 24-48 hours of detachment; certain epithelial cells, notably rat intestinal epithelial cells, perish within a few hours following substratum detachment $[9,14]$.

This paper presents current understanding of exfoliation along with the influence of methodology on the isolation of exfoliated gut epithelial cell phenotypes and, finally, 


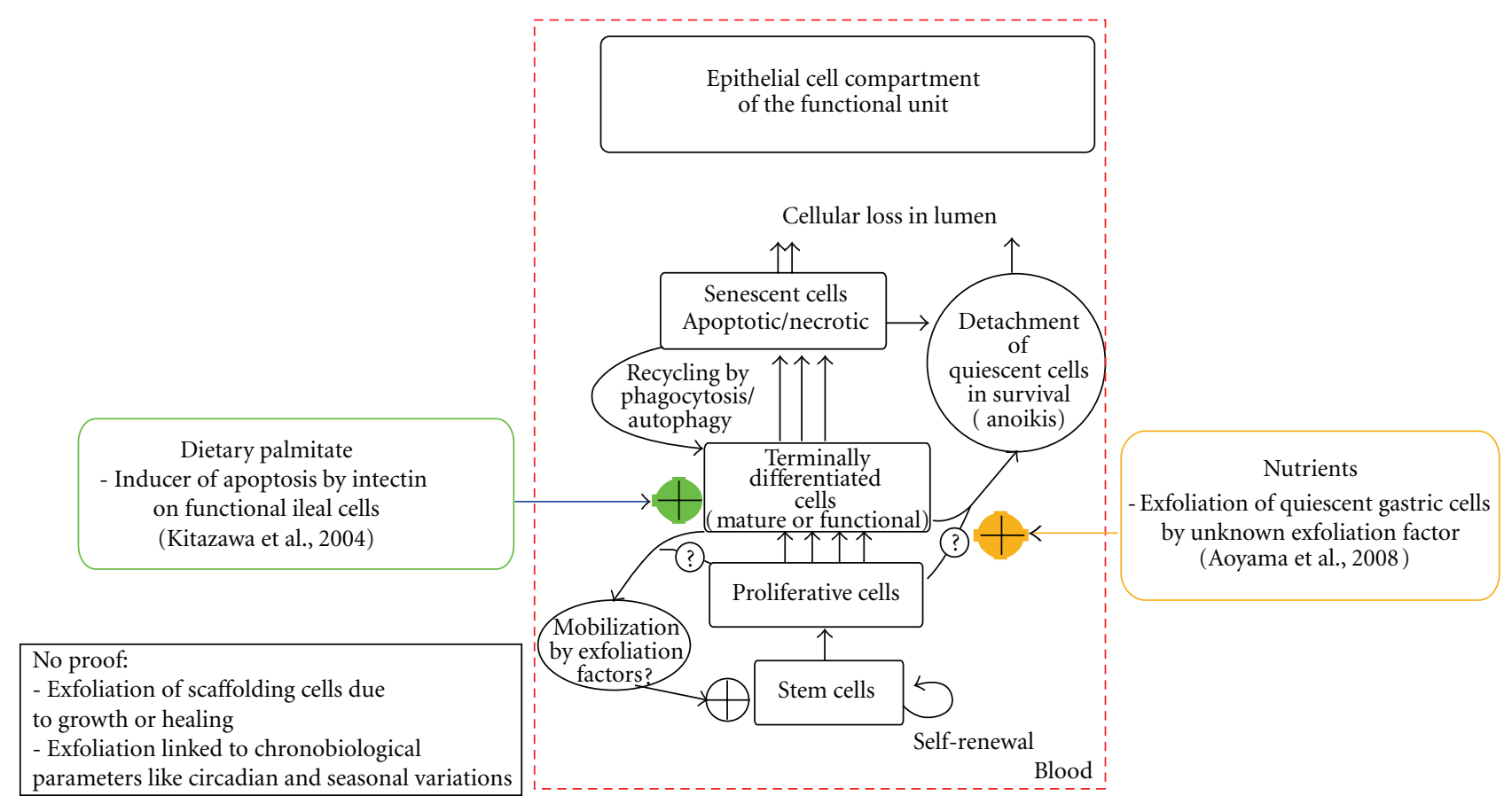

Figure 1: The concept of epithelial functional unit. The flow chart summarizes the cellular status of epithelial cells recovered by dietary palmitate on intestinal renewal (left panel) or by cycles of fasting/refeeding on gastric exfoliation (right panel). As shown in central panel, epithelial cell functional units are organized in four compartments (stem cells, proliferative, terminally differentiated, senescent). The isolation of exfoliated cells is dependent on the techniques (manual or induced by some stress), the epithelium itself, the health status and the species. A major difference in the autophagy/phagocytosis process of senescent cells has been found between rat or mouse-exfoliated colonocytes which are lost in the lumen and human colonocytes which are rarely lost in the lumen but actively recycled by neighboring cells [15]. Quiescent epithelial cells can be isolated from different body fluids (breast milk, urines, and digestives fluids). They are believed to enter rapidly in anoikis after exfoliation. Anoikis is considered as an autophagic state promoting epithelial cell survival after a timely loss of contact with extracellular matrix and cell neighbors [16]. Primary cultures can be derived from breast milk indicating that some phenotypes retain clonogenic potentials. Molecular components of circadian clocks are detected in these cells like in any other somatic cells and could provide molecular data on the expression ratios between these proteins provided sampling procedures are standardized. Further work is needed to check for any different rates of exfoliation according to the time of day, seasons, and to differences in phenotypes.

speculates on the balance between anoikis and apoptosis to explain the survival of epithelial gut cells in the environment.

\section{Exfoliation of Epithelial Cells: A Source of Reliable Biological Information on the Mucosa Physiology?}

Exfoliation can be understood as a natural process to preserve tissue architecture. Following that first point of view, exfoliation is a loss of cellular material retaining the basic cytological features of typical cells (plasma membrane, cytoplasm, and nucleus). Exfoliated epithelial cells can be obtained from a wide range of mucosae whose line body passages and cavities communicating directly or indirectly with the exterior like mammary glands, oral, bronchial, urothelial, or gastrointestinal epithelia. Epithelia can be classified as simple cylindrical cell monolayers like colon or pseudostratified like urothelium [17]. According to histology, epithelia are organized in functional units containing different cellular compartments (stem, proliferative, mature, or functional and senescent) as shown in Figure 1. These functional units are always at the interface with the environment. At a given time point, a mucosal epithelium is supposed to loose different categories of cells by different mechanisms of exfoliation. However, the cell turnover of these epithelial cells is driven by a delicate balance between cellular loss and proliferation. Proliferation is running on two cellular compartments, the proliferative cells capable of rapid mitosis to amplify tissue regeneration and the stem cells which are giving rise to all phenotypes by asymmetric mitosis. The speed of mitosis in proliferative compartment is dependent on cellular loss at the top of the structure and on tightly regulated cell migration along the functional units [18]. Cell migration in the small and large bowels of mice shows a strong circadian rhythm, with cell velocity maximal at 9 a.m. and minimal at 5 p.m [19]. Other rhythms which could be controlled by circadian clocks have been observed in the intestine like cellular proliferation [20,21] or apoptosis [22]. Cell proliferation is also believed to be under the control of clockwork not only in hepatocytes [23] but also along the rat's gut [24]. Seasonal rhythms of proliferation have been described in adult rats $[25,26]$. Circadian as well as seasonal rhythms in cell proliferation seem clearly relevant to the recovery of exfoliated quiescent cells retaining specific and functional biomarkers. 
However, there is a second point of view where exfoliation is a loss of cells in the environment due to external mechanical forces like brushing or friction. Such forces are deeply altering the epithelium architecture but allow to yield rapidly high amounts of epithelial cells retaining phenotypes and physiological status as close as possible to the mucosal cells remaining in the epithelium. Manual exfoliation has been reported with brushing or scraping technique on oral epithelium of cheek or tongue [27], cervical [28], or rectal swabbings [29], airway epithelial cells in sputum and buccal mucosal cells obtained by rinsing the mouth or chewinggum (betel chewers-[30]), esophageal cells [31], mammary by nipple aspirate, ductal lavage Klein et al., [12], breast milk [11], or bladder urothelial cells present in urine samples $[32,33]$. Manual exfoliation has also been proposed as a way to recover intact, normal epithelial cells on tissue biopsies made on colon resection [34, 35]. Some device has also been designed to recover surface exfoliated cells of human rectal mucosa by a minimal invasive scraping [36]. The technique partially purifies the cell preparation by taking advantage of the cell's inherent biology. Epithelial cells remained in small groups or sheets, detached from any stromal elements that may have been scraped off [34]. This observation is also true for gastric epithelial cells [4]. The problem is that in most clinical situation, there is no direct access to the mucosa and the technique simply cannot be used.

Consequently, we may wonder whether some useful biological information can be recorded from relatively low numbers of cells, isolated as a mixture of cellular phenotypes with different physiological states? Magnetic beads and antibodies are well-known systems to recover low numbers of epithelial cells in biological fluids or to recover highly purified epithelial phenotypes. Antibodies against human cell surface antigens like anti-Hep [48], or antiepithelial Surface marker [4] from an original antibody described by Moldenhauer et al., [49] or anti-Ber-Ep4 [34,35] from an original antibody described by Sheibani et al. [50] labeled with paramagnetic particles are used to capture and to purify epithelial cells. Viability of recovered cells by this exfoliation/enrichment method as well as by other similar techniques is on average between 90 and $100 \%$ by the Trypan blue exclusion assay. However, in our experience, shieving is necessary to perform immunocapture. With samples containing sheets of 5 to 30 cells, shieving or Percoll gradients are not adapted as this precious cellular material is discarded by the purification process. In addition, microbial contaminations are not easily removed by such density gradient methods because microbes are tightly associated with cells. Even with the manual exfoliation technique [35], the exfoliated cell populations may contain other cell types, most notably lymphocytes and plasma cells. It should be underlined that the problem of crosscontaminations by other sources of exfoliated epithelial cells like breast cells from the milk with gastric cells of lactating infants or exfoliated cells from manipulators is particularly difficult to ward off calling for the development of biomarkers of tissue origin which can guarantee both the cellular origin and the affordability of testing.

The next section discusses recent works in 3D mammary reconstruction and the functioning of acini which have shed new light on the capacity of surface epithelial cells to survive outside their epithelium. Laboratory rodent models are also discussed as they open the possibility to induce exfoliation by nutritional manipulations.

\section{In Vitro and Animal Model to Study Exfoliation}

3.1. In Vitro Model to Study the Loss of Contact of Epithelial Cells with Extracellular Matrix and Cellular Neighbors. Exfoliated epithelial cells are in a situation similar to freshly isolated primary cells. Primary cells are inoculated as singlecell suspensions or small clumps of cellular aggregates. These cells have also lost contact with the tissue architecture (companion fibroblasts, epithelial cell neighbors, and with the extracellular matrix), as well as with the nervous regulation or the blood nutriments. From tissue cultures, we know that a molecule of nutriment has to be within $50 \mathrm{~nm}$ away from a single cell to be accessible. So even if some cells are exfoliated in a nutritious matrix (milk for instance), they may have to trigger a survival mechanism. Some set of genes are progressively turned-down like clock genes [51], but in this particular situation, they can be reinduced under specific stimulation in culture. Over the years, the conditions of culture have been adapted to mimic the tissue architecture by creating three-dimensional (3D) environment.

Recent works have shown that autophagy can be observed during lumen formation in $3 \mathrm{D}$ cell cultures in vitro. The MCF-10A cells are a nontransformed human mammary epithelial cell line, which can form spherical structures (called acini) in which a layer of polarized epithelial cells surrounds a hollow lumen, mimicking the glandular epithelium in vivo $[8,9]$. The lesson we can learn from this $3 \mathrm{D}$ reconstruction of mammary gland is that epithelial cells are able to flexibly leave or reenter an epithelium. The property is useful for tissular growth as well as to heal rapidly microlesion in the epithelium cell lining. Epithelial cells exfoliated in milk are able to withstand lumen environment. Their biochemical state should be close to the state of cells having lost contact with the $3 \mathrm{D}$ reconstructed gland. In the next section, we present recent works in laboratory rodents which have shown that this capacity of an epithelial cell to adapt to changing environmental conditions is highly context dependent.

3.2. Animal Models to Study Exfoliation. Laboratory rodent models have been developed to study the inducing effect of nutrient intake on the exfoliation of epithelial cells in the digestive lumen. On adult rats fastened for 24 hours and refed for one hour, the feeding intake induces exfoliation of quiescent parietal cells at the top of the gastric gland through an unknown exfoliation factor [5]. Under these conditions, stem cells located in the neck region of gastric glands are believed to be recruited actively to repopulate the surface of the adult rat stomach. On lactating rat pups, we obtained similar results by fastening the pups for 5 hours and allowed them to be reunited with their mother for one hour before sacrifice [43]. By contrast, on adult laboratory mice, fatty acids (like palmitate) are inducers of intectin, a protein 
implicated in the exfoliation of apoptotic cells at the top of the villus of the small intestine within an hour after meal [3]. These models of nutritional manipulations to induce exfoliation on small intestine of mice [3] or gastric mucosa of rats [5] indicate that the mechanism of exfoliation is highly context dependent, but they also open the possibility to develop in vivo studies of anoikis and autophagy in relation with the functioning of peripheral circadian clocks. The disponibility of laboratory rodent models is of paramount importance to develop in vivo studies on anoikis and its connection with molecular circadian clocks to evaluate the stability of chronobiological molecular information in exfoliated cells. The proof that exfoliation of quiescent cells is following a circadian rhythm is still missing, probably because the set of physiological parameters leading to the induction of active exfoliation is difficult to handle and the interpretation of data obtained from manual exfoliation is also highly context-sensitive.

In conclusion, exfoliation is a broad term recovering many different biological or experimental situations but as illustrated by the next section, progress in the understanding of the delicate balance between autophagy and apoptosis will help scientists to design new bioassays tailored for specific clinical situations.

\section{Stability of Biological Information in Exfoliated Epithelial Cells}

Exfoliated cancerous cells of epithelial origin have been the first to be used to help design noninvasive screening assay of cancerous patients $[52,53]$. The relatively high loss of cancerous epithelial cells by patients as well as the stability of molecular information (genetic alterations related to colon cancer, for instance) have helped to establish the methodology.

Recently, Chapkin et al. [6] have developed and patented a transcriptomic approach to explore exfoliation in stools of infants as well as in adults [6]. The weak point of this approach is that the morphological information of the cell population is lost during the extraction process of mRNA, and there is no possibility to check for the exact cellular origin of these molecules. Exfoliation in stools is still highly debated; some visual proof of typical intestinal cells have been published $[4,48]$, but in my experience if whole crypt material or typical colonocytes can be found, most of the time the criticism of Loktionov [29] that these cells cannot be distinguished from epithelial cells of the anal zone is correct. The proof of similarity between exfoliated epithelial cells with the ones remaining in the mucosa will be probably easier to perform on gastric epithelium following the seminal work of Aoyama et al. [5]. However, the detection of proteins and structural elements will remain possible only in a limited number of clinical situations narrowing the possibility of using exfoliated epithelial cells as indicator of good health.

Most biological information is highly labile and is rapidly lost after exfoliation. However, a better understanding of the key factors allowing the cellular survival outside the tissue architecture will open new avenues to derive useful screening assays from clinical material. The detachment of epithelial cells from the tissue architecture triggers both pro- and antiapoptotic signals, such as nuclear factor kappa-B and inhibitor of apoptosis protein family members; these antiapoptotic mechanisms presumably delay the onset of apoptosis and allow cells to survive [54-56]. The balance between these signals and the duration of detachment determine further fate of these cells. Antiapoptotic signals presumably delay the onset of anoikis, allowing cells to survive provided that they can reestablish extracellular matrix contact in a timely manner [16]. In cells having lost contact with tissular structure, autophagy corresponds to the recycling of cellular material as well as to the cell capacity to mobilize reserves during periods of starvation. Autophagy is a biochemical pathway allowing survival during fasting period which can be stopped at the organism level to prevent self-digestion. There are three main forms of autophagy: microautophagy, macroautophagy, and chaperone-mediated autophagy [57-59]. In macroautophagy, a portion of the cytosol or organelles are sequestered in a double-membrane-bound vesicle, the autophagosome (Figures 2(a) and 2(b)). A core molecule in autophagy regulation is the kinase mammalian target of rapamycin (mTOR). By sensing signals that monitor nutrient levels, mTOR can trigger protein translation by specific phosphorylation of the ribosomal protein S6 kinase (pS6K) [60].

Recent works on the molecular pathway regulating microtubule-associated protein light chain $3 \mathrm{~b}$ (LC3b) and autophagy [38] support the idea that regulation of autophagy is interconnected with regulation of apoptosis. LC3b may regulate the extrinsic apoptosis pathway in the lung through direct interactions with caveolin-1 and Fas [61]. Implication of Beclin-2 modifying factor and the antiapoptotic proteins of the Bcl-2 family in the anoikis process have been proposed to play a central role in the survival of human intestinal epithelial cells [62]; this work partly explains, at the molecular level, the low survival rate of exfoliated epithelial intestinal cells. However, autophagy has been demonstrated to occur in vivo in the surface epithelial cells of neonatal small intestine of piglets [63]. In contrast with the data of Hausmann et al. [62], the reports of Nair et al. [64] and Chandel et al. [65] are indicating that high amount of living colonocytes can be recovered from stools $\left(5 \times 10^{4}\right.$ $2 \times 10^{6}$ cells/g of stool). From a physiological point of view in infants as well as in adults, some epithelial cells may survive in a state of macroautophagy close to the surface of the epithelium up to finding their way back in the cellular lining. A device can then easily remove such cells (i.e., exfoliate these cells) by mechanical forces. In theory, the physiological status and the genetic profile of these epithelial cells should be close to the ones at the surface of the mucosa.

At the intracellular level, autophagosomes are connected to mTOR and CLOCK pathways, and if needed to apoptotic pathways. Autophagy can be described in amino-acid-free situations (Figure 2(a)) as well as in glucose-free situations (Figure 2(b)). The balance is specially relevant in protein kinetics in preterm infants where amino acids are provided by intravenous solutions [66]. The autophagic stage is probably deeply affecting the cellular metabolism. In mammals, autophagy undergoes rhythmic variation in accordance with 


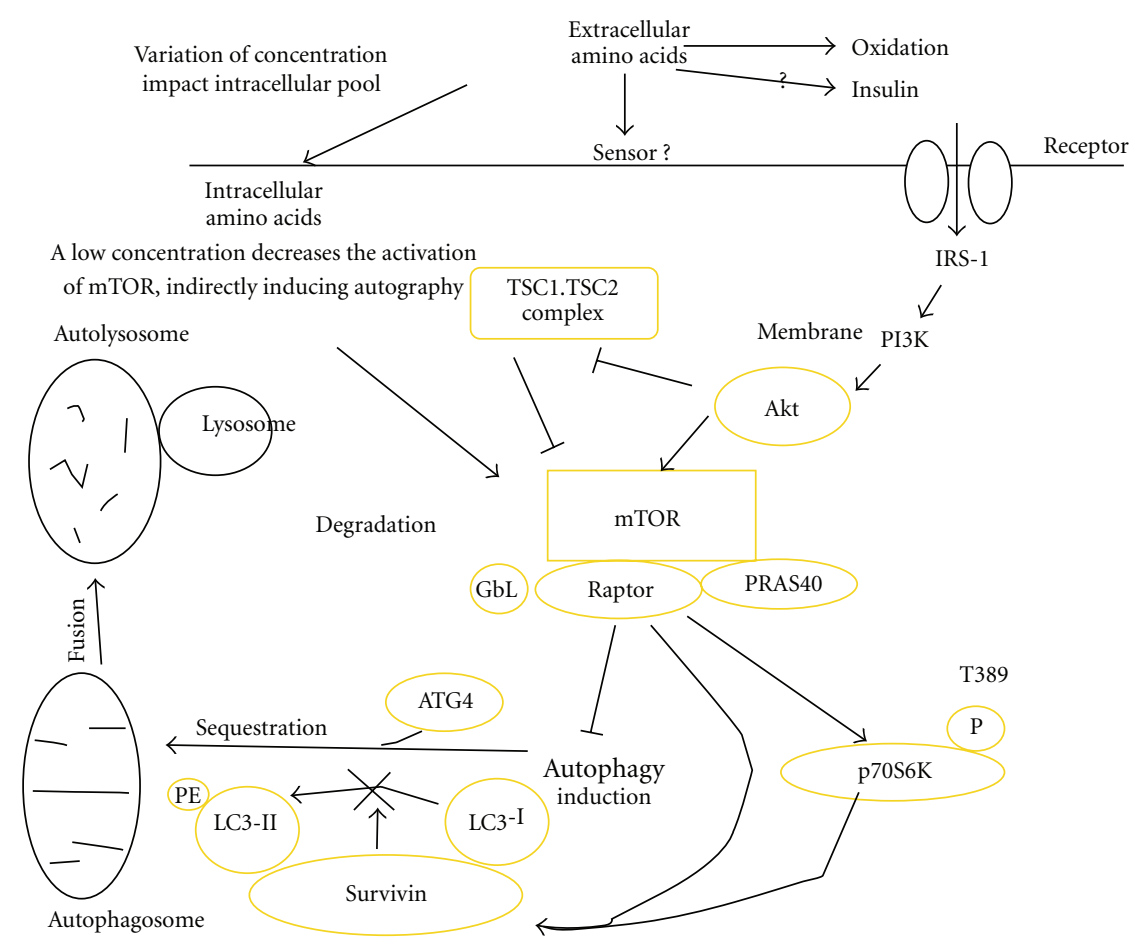

(a) Amino-acid-free conditions

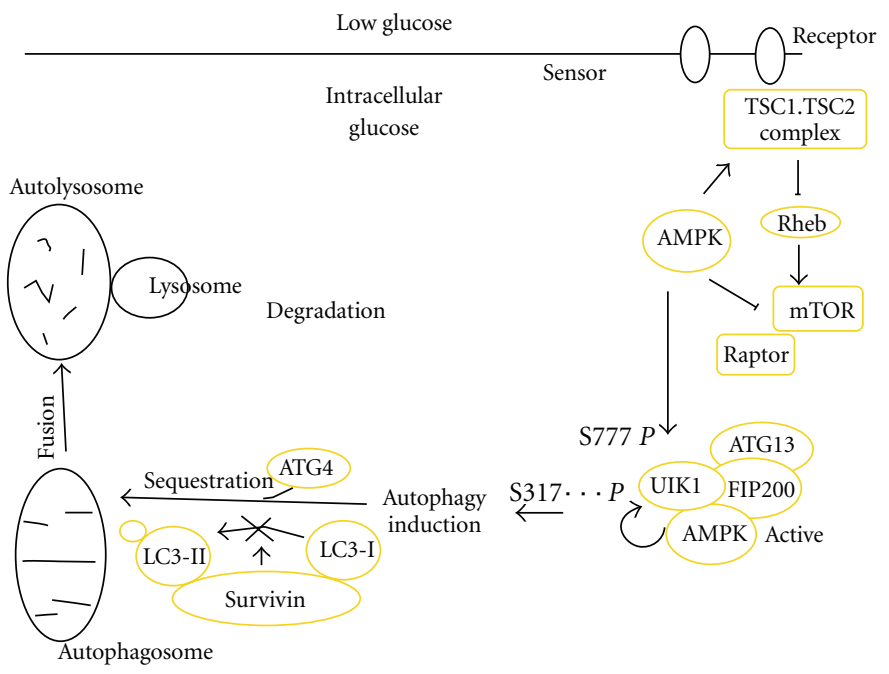

(b) Glucose-free conditions

FIGURE 2: From a physiological point of view, exfoliated epithelial cells resulting from nutritional induction or mild stress are in anoikis by activating a survival mechanism partly using the molecular paths of macroautophagy. Macroautophagy, here referred to as autophagy has been described in amino-acid-free (a) as well as in glucose-free (b) situations. Autophagosome elongation is triggered by lipid modification of LC3 (by phosphatidylethanolamine, PE). The first pathway is linked to growth factors and nutrient-sensing pathways (a), and the second is related to energy-sensing pathway (b). Detachment of extracellular matrix induces both pathways and may activate also the integrated stress response through PERK and eIF2alpha, [16] as such the situation of exfoliated cells is complex and needs more biochemical description to delineate stable molecular tags, useful in assay design, from the labile ones. The experimental starvation of primary or cancerous cells in culture consists in exposing cells to eagle minimum essential medium without serum for few hours. Under these conditions, amino-acid-free situations have been described [37, 38]. Phosphorylation involving Ulk1 in AMPK regulation has been demonstrated [39]. Survivin has also been involved in the inhibition of the conversion of LC3-I to LC3-II form (i.e., acting as an inhibitor of autophagosome formation-[40]) but in cancer cells or stem cells. However, the role of survivin in the turnover of adult cells is still debated [41, 42]. The expression of survivin has been reported in gastric parietal cells both in adult rat and human [38]. Gastric exfoliated epithelial cells of preterm infants do express high amount of survivin suggesting a crucial role of this molecule in the survival of these cells [43, 44]. Glucose starvation occurs at birth $[45,46]$. Along with cytokines and drugs, nutritional factors are now considered to be able to alter the balance between cellular survival and death [47]. 
the feeding cycles. The relationship between autophagy and circadian rhythms has been proposed [67], but the molecular link between these two phenomena is not yet known. Cells have to process diverse signals such as temperature, $\mathrm{pH}$, and nutrient concentrations in order to maintain a normal physiology. In vivo, cells are believed to use clock genes to organize and adapt cellular metabolisms and coordinate three-dimensional macromolecular organization (their phenotype) in a noisy molecular environment (molecular signals criss-crossing in and between cells and irrelevant chemical messages). Exfoliated epithelial cells in anoikis can be seen as a way to obtain chronobiological information dating back to the time of cells leaving the top of the functional units. Signals encoded in the amplitude domain are predominantly based on concentrations of signaling molecules, a parameter difficult to measure on exfoliated cells in anoikis without proper normalization related to the single cell level on highly purified cellular phenotypes. In addition, some highly labile biochemical modifications like phosphorylation are probably lost during the storage of biological fluids and in the isolation process or can be made irrelevant to the pathophysiology of the mucosa due to the turnover of the signal by the cellular machinery. However, addition of phosphatase inhibitors to cell extracts may preserve period proteins [68]. Significant improvements in the quality of cellular extracts may be achieved by using extraction buffers suitable to preserve clock gene products. The biological information encoded in the frequency domain of an oscillatory signal can be transmitted as concatenated signals with multiple biologically significant signals to gene behind the regulatory sequence within the promoter. On transgenic mammals, oscillations can be measured by recording rhythms of light emissions by cells in which the promoter of some clock gene is linked to a luciferase reporter. On human or nontransgenic mammals, oscillations per se cannot be measured in absence of spectrofluorimetric methods applicable on freshly recovered living cells, but indirect evidence of gene-circuit activation may be recovered. Long-lasting or resilient information may be accessible either through (1) the machinery of transcription at the site of fixation on the DNA of the cells or (2) by the histone code as these epigenetics modifications are believed to be acquired with a stability related to the original tissue ([69] and to the time of day [70]).

The chronobiological information that we can extract from exfoliated epithelial cells depends on the techniques used to isolate cellular material or the manipulation of physiological parameters (Figure 1) and on the affinity of interactions of clock molecular components with stable molecules like DNA or the persistence of the physiological effects that they are inducing. We may speculate that quiescent cells like epithelial gastric cells are retaining fully functional clocks, that is, consistent information with their time at exfoliation and subsequent cell survival out of the organism. The induction of gastric cell exfoliation by nutrient cycle developed in rats [5] that we have adapted on lactating rat pups [43] can be used in the future to address questions about the stability of clock information during anoikis. Exfoliated epithelial cells can be followed by microscopic examination from the initial step of loss of contact at the mouth of the gastric gland to the recovery of cells in the stomach lumen. In addition, the model is clearly relevant to clinical situation in which patients are equipped with nasogastric tubing. However, there may be tissue-specific differences in the molecular composition of the circadian clock, and clock components that have subtle effects on the central clock function may play a more prominent role in the regulation of peripheral clocks. Yagita et al. [71] have used spontaneously immortalized mouse embryo fibroblasts to explore the main clock components (proteins and mRNA) suggesting that peripheral clocks in cultured cells may be similar in composition and regulation as central clock, but all these components are not always present in cells depending on their tissular origin. The most striking example is the apparent redundancy of clock with its homolog npas2, which are largely equivalent molecules with strict structural differences $[72,73]$. Exfoliated epithelial cells after loosing contact with extracellular matrix are surviving by activating autophagy. Three main physiological pathways have been described associated to the regulation of autophagy: AKT (energy sensing), EGR-R (growth factor sensing), and bcl-2 (stressrelated programmed-cell death). According to Gan et al. [74], mTOR downregulation is also observed following extracellular matrix detachment. Clock components are probably also altered during this process, but there are no data on the relation between autophagy and clock regulation.

By contrast, explants of tissue isolated from transgenic rat for period1 gene is giving some chronobiological information about the chaotic expression of this gene under the drastic external conditions of explantation [75]. Recording of luminescence emitted in vitro by the explants maintained in classic tissue culture conditions $\left(37^{\circ} \mathrm{C}, 5 \% \mathrm{CO}_{2}\right)$ has clearly shown that the chaotic light emissions by the transgene system stands up to 12-14 hours, thereafter the rhythms of light emissions by liver explants are organized according to an oscillatory model reminiscent of period1's in vivo oscillations (as of Stokkan et al., [75], the phase of the peak has been recorded during the first subjective day in culture i.e., between 12 and 36 hours). To avoid such chaotic evolution with the loss of chronobiological information, experimenters are using mechanical punches of mucosae which are directly snap frozen in liquid nitrogen. This strategy is a reliable but invasive solution to study clock gene expression in time series. Otherwise, tissue biopsies can be explanted in culture to derive primary cells or cell lines and record clocks functioning just like with exfoliated cells. However, cell lines have lost contact with body's network, and their clock systems are probably quickly reorganized to tune up with their new in vitro environment $[76,77]$. In the future, the use of transgenic mice for autophagic gene circuitry will also help to appreciate the exfoliation status and the molecular link between clocks and autophagy [78].

\section{Perspectives}

The development of non-invasive methods is crucial to allow easy sampling of human populations in nutritional/clinical intervention studies. Exfoliated epithelial cells could be 
extremely useful to deal with subtle environmental influences during development causing persistent changes in epigenetic regulation. They represent an alternative to fibroblasts which can be easier to collect on adults, and they putatively are giving molecular information from inner tissues difficult to access. The problem in the recovery of exfoliated cells is to relate the cell phenotypes and their physiological status with the intact tissue structure of the donor. In addition, the degradation of biological information depends on the bioactive compounds present in the biological fluids which can be heavily loaded with enzymes like digestive fluids.

A specific application explored in our laboratory is to develop studies in exfoliation in order to improve nursing care in preterm infants and to prevent the onset of such a syndrome during adult life. Tracking exfoliated epithelial cells can be used to follow the renewal of the gastric epithelium in order to monitor nutritional or pharmacological practices. Recently, we have shown that circadian clock genes were disregulated following an episode of perinatal denutrition [79]. The isolation of exfoliated epithelial cells from pups or infants suffering from perinatal denutrition at the onset of the problem or later in adult life may help to know whether the histone acetyltransferase's activity of CLOCK can be used to explore the stability of epigenetic profile in exfoliated epithelial cells. However, there is a lack of biomarkers to study exfoliated epithelial cells and the role of clock genes, if any, in autophagy. Among many unsolved questions which can be listed, we can wonder what is the biological information retained, altered, or lost during anoikis? Future works may focus on the mTOR signaling pathway which has been found downregulated in detached epithelial cells [74] and, in adipocytes, linked to diurnal gene expression and metabolic regulation [80]. Recent data on the molecular biology of clock components indicate that central and peripheral clocks differ in their coupling with the different categories of synchronizers as well as in their output on rodent models [81, 82] as well as on human data [68]. A better understanding of exfoliation may be useful not only to translational research but also to tissue reconstruction of mucosa.

\section{Acknowledgments}

The author is grateful for 2 financial supports: ANSSD-2008 from Département Alimentation Humaine (Inra, France); PremaCol-2008 (Région Pays de la Loire, France).

\section{References}

[1] C. D. Davis, "Use of exfoliated cells from target tissues to predict responses to bioactive food components," Journal of Nutrition, vol. 133, no. 6, pp. 1769-1772, 2003.

[2] A. Kamra, G. Kessie, J. H. Chen et al., "Exfoliated colonic epithelial cells: surrogate targets for evaluation of bioactive food components in cancer prevention," Journal of Nutrition, vol. 135, no. 11, pp. 2719-2722, 2005.

[3] H. Kitazawa, T. Nishihara, T. Nambu et al., "Intectin, a novel small intestine-specific glycosylphosphatidylinositolanchored protein, accelerates apoptosis of intestinal epithelial cells," Journal of Biological Chemistry, vol. 279, no. 41, pp. 42867-42874, 2004.
[4] B. Kaeffer, C. Des Robert, M. C. Alexandre-Gouabau et al., "Recovery of exfoliated cells from the gastrointestinal tract of premature infants: a new tool to perform "noninvasive biopsies?"'” Pediatric Research, vol. 62, no. 5, pp. 564-569, 2007.

[5] F. Aoyama, A. Sawaguchi, S. Ide, K. Kitamura, and T. Suganuma, "Exfoliation of gastric pit-parietal cells into the gastric lumen associated with a stimulation of isolated rat gastric mucosa in vitro: a morphological study by the application of cryotechniques," Histochemistry and Cell Biology, vol. 129, no. 6, pp. 785-793, 2008.

[6] R. S. Chapkin, C. Zhao, I. Ivanov et al., "Noninvasive stool-based detection of infant gastrointestinal development using gene expression profiles from exfoliated epithelial cells," American Journal of Physiology, vol. 298, no. 5, pp. G582G589, 2010.

[7] S. M. Frisch and H. Francis, "Disruption of epithelial cellmatrix interactions induces apoptosis," Journal of Cell Biology, vol. 124, no. 4, pp. 619-626, 1994.

[8] J. Debnath, K. R. Mills, N. L. Collins, M. J. Reginato, S. K. Muthuswamy, and J. S. Brugge, "The role of apoptosis in creating and maintaining luminal space within normal and oncogene-expressing mammary acini," Cell, vol. 111, no. 1, pp. 29-40, 2002.

[9] C. Fung, R. Lock, S. Gao, E. Salas, and J. Debnath, "Induction of autophagy during extracellular matrix detachment promotes cell survival," Molecular Biology of the Cell, vol. 19, no. 3, pp. 797-806, 2008.

[10] B. Levine and D. J. Klionsky, "Development by self-digestion: molecular mechanisms and biological functions of autophagy," Developmental Cell, vol. 6, no. 4, pp. 463-477, 2004.

[11] P. A. Thompson, F. F. Kadlubar, S. M. Vena et al., "Exfoliated ductal epithelial cells in human breast milk: a source of target tissue DNA for molecular epidemiologic studies of breast cancer," Cancer Epidemiology Biomarkers and Prevention, vol. 7, no. 1, pp. 37-42, 1998.

[12] P. Klein, E. Glaser, L. Grogan et al., "Biomarker assays in nipple aspirate fluid," Breast Journal, vol. 7, no. 6, pp. 378-387, 2001.

[13] L. A. Davidson, J. R. Lupton, E. Miskovsky, A. P. Fields, and R. S. Chapkin, "Quantification of human intestinal gene expression profiles using exfoliated colonocytes: a pilot study," Biomarkers, vol. 8, no. 1, pp. 51-61, 2003.

[14] B. Kaeffer, "Mammalian intestinal epithelial cells in primary culture: a mini-review," In Vitro Cellular and Developmental Biology, vol. 38, no. 3, pp. 123-134, 2002.

[15] E. M. M. Van Lieshout, W. Van Doesburg, and R. Van Der Meer, "Real-time PCR of host DNA in feces to study differential exfoliation of colonocytes between rats and humans," Scandinavian Journal of Gastroenterology, vol. 39, no. 9, pp. 852-857, 2004.

[16] R. Lock and J. Debnath, "Extracellular matrix regulation of autophagy," Current Opinion in Cell Biology, vol. 20, no. 5, pp. 583-588, 2008.

[17] S. M. Karam, "Lineage commitment and maturation of epithelial cells in the gut," Frontiers in Bioscience, vol. 4, pp. D286298, 1999.

[18] C. S. Potten and M. Loeffler, "Stem cells: attributes, cycles, spirals, pitfalls and uncertainties. Lessons for and from the crypt," Development, vol. 110, no. 4, pp. 1001-1020, 1990.

[19] J. M. Qiu, S. A. Roberts, and C. S. Potten, "Cell migration in the small and large bowel shows a strong circadian rhythm," Epithelial Cell Biology, vol. 3, no. 4, pp. 137-148, 1994.

[20] K. N. Buchi, J. G. Moore, W. J. M. Hrushesky, R. B. Sothern, and N. H. Rubin, "Circadian rhythm of cellular proliferation 
in the human rectal mucosa," Gastroenterology, vol. 101, no. 2, pp. 410-415, 1991.

[21] G. Marra, M. Anti, A. Percesepe et al., "Circadian variations of epithelial cell proliferation in human rectal crypts," Gastroenterology, vol. 106, no. 4, pp. 982-987, 1994.

[22] R. Iwakiri, Y. Gotoh, T. Noda et al., "Programmed cell death in rat intestine: effect of feeding and fasting," Scandinavian Journal of Gastroenterology, vol. 36, no. 1, pp. 39-47, 2001.

[23] T. Matsuo, S. Yamaguchi, S. Mitsui, A. Emi, F. Shimoda, and H. Okamura, "Control mechanism of the circadian clock for timing of cell division in vivo," Science, vol. 302, no. 5643, pp. 255-259, 2003.

[24] L. Polidarová, M. Soták, M. Sládek, J. Pácha, and A. Sumová, "Temporal gradient in the clock gene and cell-cycle checkpoint kinase wee1 expression along the gut," Chronobiology International, vol. 26, no. 4, pp. 607-620, 2009.

[25] E. Haus, D. J. Lakatua, L. Sackett-Lundeen, and M. White, "Circannual variation of intestinal cell proliferation in BDF1 male mice on three lighting regimens," Chronobiology International, vol. 1, no. 3, pp. 185-194, 1984.

[26] J. R. Gomes, A. A. M. Pereira, L. Barth et al., "Circadian variation of the cell proliferation in the jejunal epithelium of rats at weaning phase," Cell Proliferation, vol. 38, no. 3, pp. 147-152, 2005.

[27] N. Holland, P. Harmatz, D. Golden et al., "Cytogenetic damage in blood lymphocytes and exfoliated epithelial cells of children with inflammatory bowel disease," Pediatric Research, vol. 61, no. 2, pp. 209-214, 2007.

[28] M. Steinau, D. R. Lee, M. S. Rajeevan, S. D. Vernon, M. T. Ruffin, and E. R. Unger, "Gene expression profile of cervical tissue compared to exfoliated cells: impact on biomarker discovery," BMC Genomics, vol. 1, article 64, 2005.

[29] A. Loktionov, "Cell exfoliation in the human colon: myth, reality and implications for colorectal cancer screening," International Journal of Cancer, vol. 120, no. 11, pp. 2281-2289, 2007.

[30] H. F. Stich, W. Stich, M. P. Rosin, and M. O. Vallejera, "Use of the micronucleus test to monitor the effect of vitamin A, betacarotene and canthaxanthin on the buccal mucosa of betel nut/tobacco chewers," International Journal of Cancer, vol. 34, no. 6, pp. 745-750, 1984.

[31] N. Munoz, M. Hayashi, L. J. Bang, J. Wahrendorf, M. Crespi, and F. X. Bosch, "Effects of riboflavin, retinol, and zinc on micronuclei of buccal mucosa and of esophagus: a randomized double-blind intervention study in China," Journal of the $\mathrm{Na}$ tional Cancer Institute, vol. 79, no. 4, pp. 687-691, 1987.

[32] J. S. Felix and J. W. Littlefield, "Human newborn urine as a source of epithelial cells," Birth Defects, vol. 16, no. 2, pp. 231237, 1980.

[33] N. Robine, J. P. Relier, and S. Le Bars, "Urocytogram, an index of maturity in premature infants," Biology of the Neonate, vol. 54, no. 2, pp. 93-99, 1988.

[34] W. D. Mojica, A. V. Rapkiewicz, L. A. Liotta, and V. Espina, "Manual exfoliation of fresh tissue obviates the need for frozen sections for molecular profiling," Cancer, vol. 105, no. 6, pp. 483-491, 2005.

[35] W. D. Mojica, L. Stein, and L. Hawthorn, "An exfoliation and enrichment strategy results in improved transcriptional profiles when compared to matched formalin fixed samples," BMC Clinical Pathology, vol. 7, article 7, 2007.

[36] A. Loktionov, T. Bandatelova, A. Llewelyn, C. Ferrett, R. C. G. Lywood, and H. G. G. Lywood, "Colorectal cell sampling device," World Intellectual Property Organisation. International Publication Number WO 2006/003447 A1.
[37] W. Martinet, G. R. Y. De Meyer, L. Andries, A. G. Herman, and M. M. Kockx, "Detection of autophagy in tissue by standard immunohistochemistry: possibilities and limitations," Autophagy, vol. 2, no. 1, pp. 55-57, 2006.

[38] S.-K. Chiou, N. Hoa, and A. Hodges, "Sulindac sulfide induces autophagic death in gastric epithelial cells via Survivin downregulation: a mechanism of NSAIDs-induced gastric injury," Biochemical Pharmacology, vol. 81, no. 11, pp. 1317-1323, 2011.

[39] J. Kim, M. Kundu, B. Viollet, and K. L. Guan, "AMPK and mTOR regulate autophagy through direct phosphorylation of Ulk1," Nature Cell Biology, vol. 13, no. 2, pp. 132-141, 2011.

[40] H. Roca, Z. S. Varsos, K. Mizutani, and K. J. Pienta, "CCL2, survivin and autophagy: new links with implications in human cancer," Autophagy, vol. 4, no. 7, pp. 969-971, 2008.

[41] S. Fukuda and L. M. Pelus, "Survivin, a cancer target with an emerging role in normal adult tissues," Molecular Cancer Therapeutics, vol. 5, no. 5, pp. 1087-1098, 2006.

[42] D. C. Altieri, "New wirings in the survivin networks," Oncogene, vol. 27, no. 48, pp. 6276-6284, 2008.

[43] B. Kaeffer, H. Qi, R. J. B. Matos et al., "Exfoliated epithelial cells, a source of information on clock genes expression by preterm infants to explore the onset of metabolic syndrome," in Proceedings of the 11th Congress of the European Biological Rhythms Society, Strasbourg, France, August 2009.

[44] B. Kaeffer and J.-C. Rozé, "Procédé d'évaluation in vitro de l'état physiologique d'une muqueuse gastrique et kit pour réaliser une telle évaluation," Brevet d'invention, FR1054245, 2010.

[45] A. Kuma, M. Hatano, M. Matsui et al., "The role of autophagy during the early neonatal starvation period," Nature, vol. 432, no. 7020, pp. 1032-1036, 2004.

[46] O. B. Kotoulas, S. A. Kalamidas, and D. J. Kondomerkos, "Glycogen autophagy in glucose homeostasis," Pathology Research and Practice, vol. 202, no. 9, pp. 631-638, 2006.

[47] W. Bursch, A. Karwan, M. Mayer et al., "Cell death and autophagy: cytokines, drugs, and nutritional factors," Toxicology, vol. 254, no. 3, pp. 147-157, 2008.

[48] T. Bandaletova, N. Bailey, S. A. Bingham, and A. Loktionov, "Isolation of exfoliated colonocytes from human stool as a new technique for colonic cytology," APMIS, vol. 110, no. 3, pp. 239-246, 2002.

[49] G. Moldenhauer, F. Momburg, P. Moller, R. Schwartz, and G. J. Hammerling, "Epithelium-specific surface glycoprotein of $\mathrm{M}(\mathrm{r}) 34,000$ is a widely distributed human carcinoma marker," British Journal of Cancer, vol. 56, no. 6, pp. 714-721, 1987.

[50] K. Sheibani, S. S. Shin, J. Kezirian, and L. M. Weiss, "BerEP4 antibody as a discriminant in the differential diagnosis of malignant mesothelioma versus adenocarcinoma," American Journal of Surgical Pathology, vol. 15, no. 8, pp. 779-784, 1991.

[51] B. Kaeffer and L. Pardini, "Clock genes of mammalian cells: practical implications in tissue culture," In Vitro Cellular and Developmental Biology, vol. 41, no. 10, pp. 311-320, 2005.

[52] N. K. Osborn and D. A. Ahlquist, "Stool screening for colorectal cancer: molecular approaches," Gastroenterology, vol. 128, no. 1, pp. 192-206, 2005.

[53] G. P. Albaugh, V. Iyengar, A. Lohani, M. Malayeri, S. Bala, and P. P. Nair, "Isolation of exfoliated colonic epithelial cells, a novel, non-invasive approach to the study of cellular markers," International Journal of Cancer, vol. 52, no. 3, pp. 347-350, 1992.

[54] A. P. Gilemore, "Anoikis," Cell Death and Differentiation, vol. 12, supplement 2, pp. 1473-1477, 2005. 
[55] S. R. Yan, R. R. Joseph, K. Rosen et al., "Activation of NF- $\kappa$ B following detachment delays apoptosis in intestinal epithelial cells," Oncogene, vol. 24, no. 43, pp. 6482-6491, 2005.

[56] Z. Liu, H. Li, X. Wu et al., "Detachment-induced upregulation of XIAP and cIAP2 delays anoikis of intestinal epithelial cells," Oncogene, vol. 25, no. 59, pp. 7680-7690, 2006.

[57] D. J. Klionsky, "Autophagy," Current Biology, vol. 15, no. 8, pp. R282-R283, 2005.

[58] A. C. Massey, S. Kaushik, and A. M. Cuervo, "Lysosomal chat maintains the balance," Autophagy, vol. 2, no. 4, pp. 325-327, 2006.

[59] F. Cecconi and B. Levine, "The role of autophagy in mammalian development: cell makeover rather than cell death," Developmental Cell, vol. 15, no. 3, pp. 344-357, 2008.

[60] E. F. C. Blommaart, J. J. F. P. Luiken, P. J. E. Blommaart, G. M. Van Woerkom, and A. J. Meijer, "Phosphorylation of ribosomal protein S6 is inhibitory for autophagy in isolated rat hepatocytes," Journal of Biological Chemistry, vol. 270, no. 5, pp. 2320-2326, 1995.

[61] A. Chen, "The circadian rhythm of expression of Bmal1 and Cry1 in peripheral blood mononuclear cells of preterm neonates," Journal of Maternal-Fetal and Neonatal Medicine, vol. 23, no. 10, pp. 1172-1175, 2010.

[62] M. Hausmann, K. Leucht, C. Ploner et al., "BCL-2 modifying factor (BMF) is a central regulator of anoikis in human intestinal epithelial cells," Journal of Biological Chemistry, vol. 286, no. 30, pp. 26533-26540, 2011.

[63] M. M. Godlewski, N. Hallay, J. B. Bierła, and R. Zabielski, "Molecular mechanism of programmed cell death in the gut epithelium of neonatal piglets," Journal of Physiology and Pharmacology, vol. 58, no. 3, pp. 97-113, 2007.

[64] P. Nair, S. Lagerholm, S. Dutta et al., "Coprocytobiology: on the nature of cellular elements from stools in the pathophysiology of colonic disease," Journal of Clinical Gastroenterology, vol. 36, no. 5, pp. S84-S93, 2003.

[65] D. S. Chandel, G. T. Braileanu, J.-H. J. Chen, H. H. Chen, and P. Panigrahi, "Live colonocytes in newborn stool: surrogates for evaluation of gut physiology and disease pathogenesis," Pediatric Research, vol. 70, no. 2, pp. 153-158, 2011.

[66] S. C. Kalhan and J. M. Edmison, "Effect of intravenous amino acids on protein kinetics in preterm infants," Current Opinion in Clinical Nutrition and Metabolic Care, vol. 10, no. 1, pp. 69 74, 2007.

[67] U. M. Sachdeva and C. B. Thompson, "Diurnal rhythms of autophagy," Autophagy, vol. 4, no. 5, pp. 581-589, 2008.

[68] M. Gallego and D. M. Virshup, "Post-translational modifications regulate the ticking of the circadian clock," Nature Reviews Molecular Cell Biology, vol. 8, no. 2, pp. 139-148, 2007.

[69] R. A. Waterland and K. B. Michels, "Epigenetic epidemiology of the developmental origins hypothesis," Annual Review of Nutrition, vol. 27, pp. 363-388, 2007.

[70] T. Alenghat, K. Meyers, S. E. Mullican et al., "Nuclear receptor corepressor and histone deacetylase 3 govern circadian metabolic physiology," Nature, vol. 456, no. 7224, pp. 997-1000, 2008.

[71] K. Yagita, F. Tamanini, G. T. J. Van der Horst, and H. Okamura, "Molecular mechanisms of the biological clock in cultured fibroblasts," Science, vol. 292, no. 5515, pp. 278-281, 2001.

[72] M. Reick, J. A. Garcia, C. Dudley, and S. L. McKnight, "NPAS2: an analog of clock operative in the mammalian forebrain," Science, vol. 293, no. 5529, pp. 506-509, 2001.

[73] J. Rutter, M. Reick, L. C. Wu, and S. L. McKnight, "Regulation of crock and NPAS2 DNA binding by the redox state of NAD cofactors," Science, vol. 293, no. 5529, pp. 510-514, 2001.
[74] B. Gan, Y. Yoo, and J. L. Guan, "Association of focal adhesion kinase with tuberous sclerosis complex 2 in the regulation of S6 kinase activation and cell growth," Journal of Biological Chemistry, vol. 281, no. 49, pp. 37321-37329, 2006.

[75] K. A. Stokkan, S. Yamazaki, H. Tei, Y. Sakaki, and M. Menaker, "Entrainment of the circadian clock in the liver by feeding," Science, vol. 291, no. 5503, pp. 490-493, 2001.

[76] E. Nagoshi, C. Saini, C. Bauer, T. Laroche, F. Naef, and U. Schibler, "Circadian gene expression in individual fibroblasts: cell-autonomous and self-sustained oscillators pass time to daughter cells," Cell, vol. 119, no. 5, pp. 693-705, 2004.

[77] S. A. Brown, F. Fleury-Olela, E. Nagoshi et al., "The period length of fibroblast circadian gene expression varies widely among human individuals," PLoS Biology, vol. 3, no. 10, article e338, p. 6, 2005.

[78] N. Mizushima, A. Yamamoto, M. Matsui, T. Yoshimori, and Y. Ohsumi, "In vivo analysis of autophagy in response to nutrient starvation using transgenic mice expressing a fluorescent autophagosome marker," Molecular Biology of the Cell, vol. 15, no. 3, pp. 1101-1111, 2004.

[79] R. Orozco-Sólis, R. J. B. Matos, S. Lopes de et al., "Perinatal nutrient restriction induces long-lasting alterations in the circadian expression pattern of genes regulating food intake and energy metabolism," International Journal of Obesity, vol. 35, pp. 990-1000, 2011.

[80] A. Loboda, B. Fine, J. Joseph et al., "Diurnal variation of the human adipose transcriptome and the link to metabolic disease," BMC Medical Genomics, vol. 2, article 7, 2009.

[81] A. Sumová, Z. Bendová, M. Sládek et al., "Setting the biological time in central and peripheral clocks during ontogenesis," FEBS Letters, vol. 580, no. 12, pp. 2836-2842, 2006.

[82] H. Guo, J. M. Brewer, M. N. Lehman, and E. L. Bittman, "Suprachiasmatic regulation of circadian rhythms of gene expression in hamster peripheral organs: effects of transplanting the pacemaker," Journal of Neuroscience, vol. 26, no. 24, pp. 64066412, 2006. 

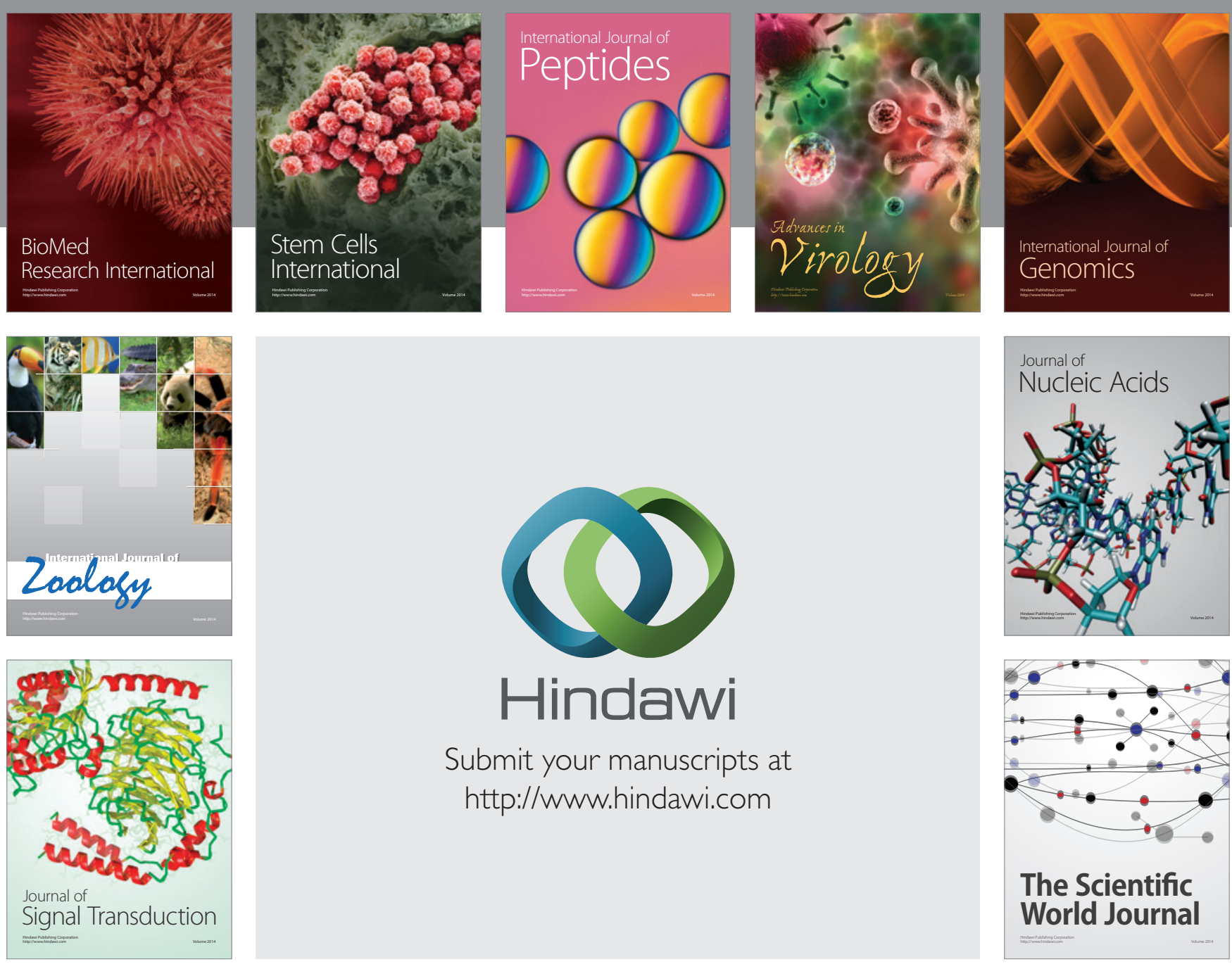

Submit your manuscripts at

http://www.hindawi.com
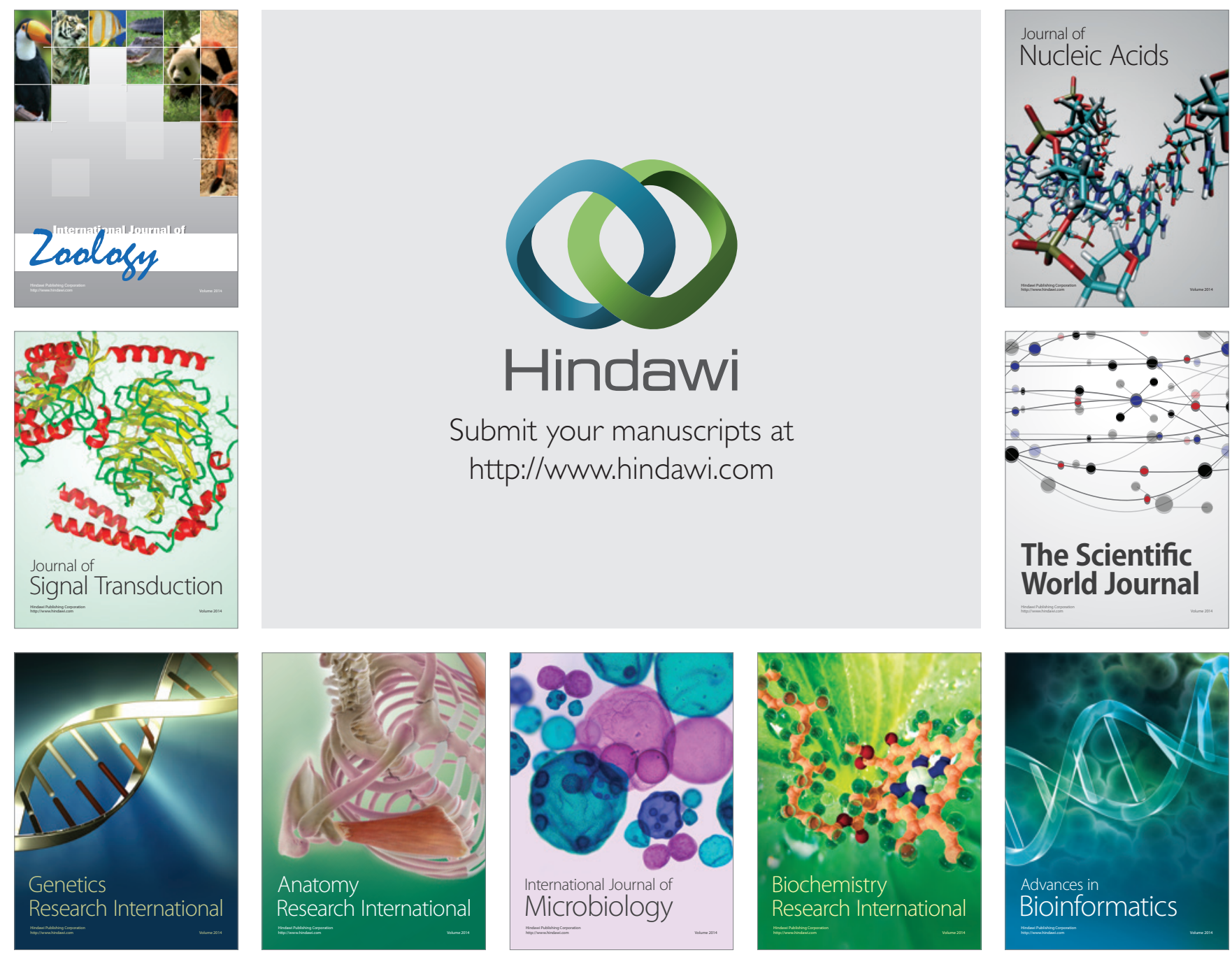

The Scientific World Journal
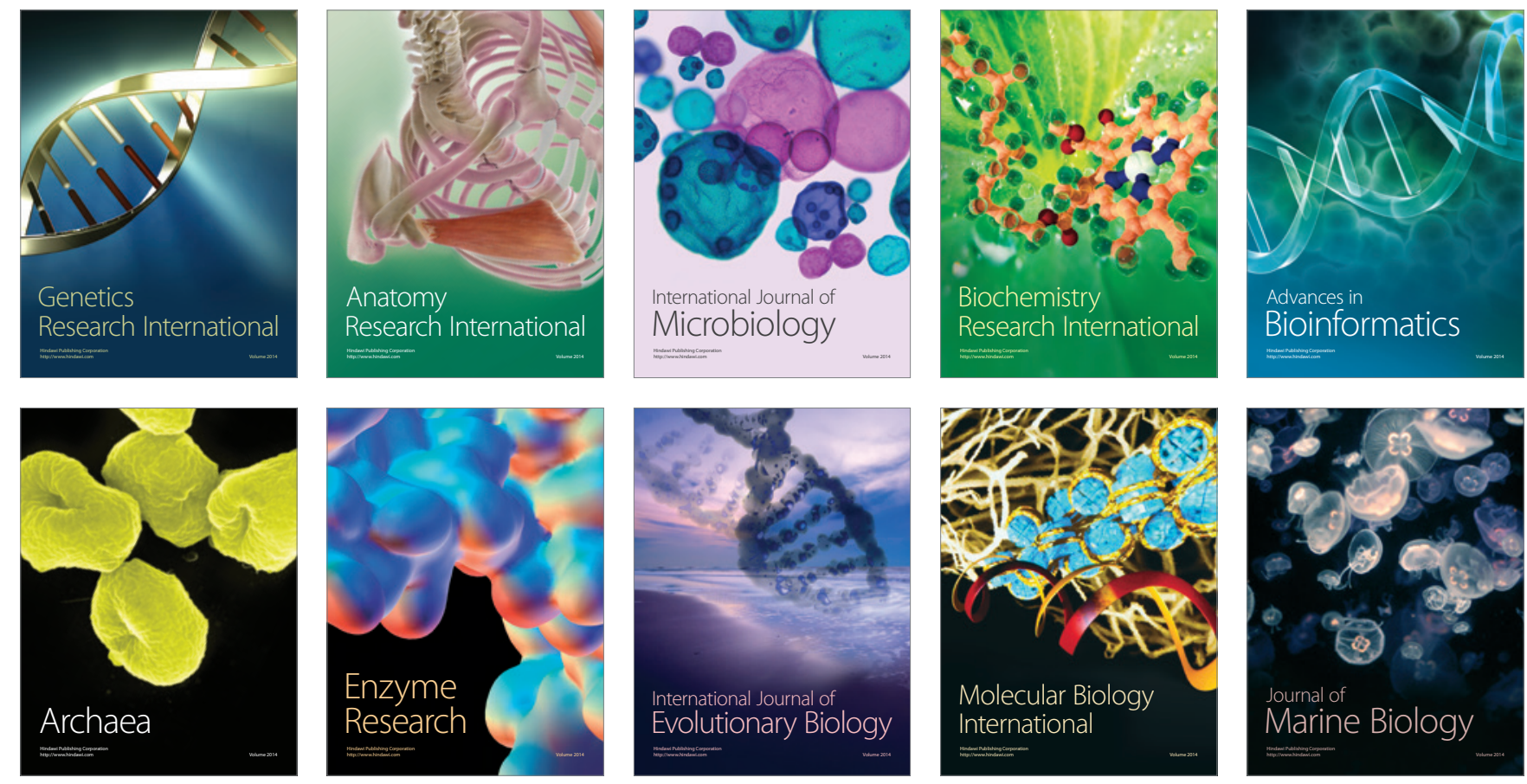